\title{
CD36 involvement in orosensory detection of dietary lipids, spontaneous fat preference, and digestive secretions
}

\author{
Fabienne Laugerette, ${ }^{1,2}$ Patricia Passilly-Degrace, ${ }^{1}$ Bruno Patris, ${ }^{3}$ Isabelle Niot, ${ }^{1}$ \\ Maria Febbraio, ${ }^{4}$ Jean-Pierre Montmayeur, ${ }^{2}$ and Philippe Besnard ${ }^{1}$
}

\begin{abstract}
1Physiologie de la Nutrition, Ecole Nationale Supérieure de Biologie Appliquée à la Nutrition et à l'Alimentation (ENSBANA) et Centre Européen des Sciences du Goût (CESG), UMR 5170 CNRS/1214 INRA/Université de Bourgogne, Dijon, France. ${ }^{2}$ Chimioréception and ${ }^{3}$ Ethologie et Psychobiologie Sensorielle, CESG, UMR 5170 CNRS/1214 INRA/Université de Bourgogne, Dijon, France. ${ }^{4}$ Cellular Biology, Lerner Research Institute, Cleveland, Ohio, USA.
\end{abstract}

\begin{abstract}
Rats and mice exhibit a spontaneous attraction for lipids. Such a behavior raises the possibility that an orosensory system is responsible for the detection of dietary lipids. The fatty acid transporter CD36 appears to be a plausible candidate for this function since it has a high affinity for long-chain fatty acids (LCFAs) and is found in lingual papillae in the rat. To explore this hypothesis further, experiments were conducted in rats and in wild-type and CD36-null mice. In mice, RT-PCR experiments with primers specific for candidate lipid-binding proteins revealed that only CD36 expression was restricted to lingual papillae although absent from the palatal papillae. Immunostaining studies showed a distribution of CD36 along the apical side of circumvallate taste bud cells. CD36 gene inactivation fully abolished the preference for LCFA-enriched solutions and solid diet observed in wild-type mice. Furthermore, in rats and wild-type mice with an esophageal ligation, deposition of unsaturated LCFAs onto the tongue led to a rapid and sustained rise in flux and protein content of pancreatobiliary secretions. These findings demonstrate that CD36 is involved in oral LCFA detection and raise the possibility that an alteration in the lingual fat perception may be linked to feeding dysregulation.
\end{abstract}

\section{Introduction}

In Western diet, about $40 \%$ of daily caloric intakes is lipid, despite the fact that the recommended level is $10 \%$ lower. This high-fat supply greatly contributes to the prevalence of obesity and associated diseases (i.e., non-insulin-dependent diabetes, atherosclerosis, and hypertension). In humans, studies showed that obese subjects prefer lipid when compared with lean subjects $(1,2)$, suggesting that inappropriate lipid perception might influence obesity risk by impacting feeding behavior.

The regulation of lipid intake is a complex phenomenon controlled by instantaneous orosensory stimuli (i.e., texture, odor, and taste) and delayed postingestive signals (3). Until recently, the involvement of gustation in this phenomenon was neglected, dietary fat being thought to be detected only by trigeminal (texture perception) and retronasal olfactory cues (4). However, short-term behavioral studies, in which normal and anosmic rodents were allowed to choose between oil- or xanthan-enriched solution (to mimic fat texture), strongly suggested that gustation plays a significant role in lipid perception $(5,6)$. Although dietary lipids consist mainly of triglycerides, compelling evidence from studies on the rat strongly suggests that long-chain fatty acids (LCFAs) may be responsible for the orosensory cue for fat. Indeed, adult animals exhibit a lower preference for triglycerides and short-chain fatty acids than for LCFAs $(6,7)$. Moreover, pharmacological inhibition of lingual lipase, the enzyme

Nonstandard abbreviations used: ACBP, acyl-CoA-binding protein; FAT, fatty acid transporter; FATP-4, fatty acid transport protein-4; I-FABP, intestinal fatty acid-binding protein; LCFA, long-chain fatty acid; L-FABP, liver fatty acid-binding protein. Conflict of interest: The authors have declared that no conflict of interest exists.

Citation for this article: J. Clin. Invest. 115:3177-3184 (2005).

doi:10.1172/JCI25299. responsible for efficient LCFA release from dietary triglycerides, profoundly decreases preference for lipids (8). Interestingly, lingual lipase level is especially high in the vicinity of taste buds, since it is locally secreted in the cleft of foliate and circumvallate papillae by the Ebner glands (8). Such an anatomical design may be sufficient to generate an LCFA stimulus in taste receptor cells. In keeping with this assumption, unsaturated LCFAs were reported to inhibit, in rat taste bud cells, the delayed rectifying $\mathrm{K}^{+}$channels known to be implicated in the transduction pathway of a variety of taste stimuli $(9,10)$. Moreover, rat lingual sensory epithelium expresses CD36 (also known as fatty acid transporter [FAT]) $(11,12)$, which binds LCFAs with an affinity in the nanomolar range $(13,14)$. The CD36 amino acid sequence predicts a ditopic glycoprotein with a large extracellular hydrophobic pocket $(15,16)$ between 2 short cytoplasmic tails. The C-terminal cytoplasmic tail has been shown to be associated with Src kinases (17), suggesting an involvement of CD36 in cell signaling. Together, these data support the existence of a chemical perception of LCFAs in the oral cavity.

Literature on the physiological advantage(s) provided by such a putative orosensory detection system is scarce. A weak rise in the protein content of pancreatobiliary juice has been reported within 10 minutes after oral delivery of LCFAs in esophagectomized rats, suggesting that the presence of lipids in the oral cavity contributes to the cephalic phase of pancreatic secretions (18). Findings demonstrate that tastant paired with fat intake can also influence lipid metabolic fate. Indeed, prolonged elevation in blood triglyceride was observed in rats in which a small amount of oil was directly administered onto the tongue before an intragastric feeding (19). Longer-term metabolic changes have also been reported in healthy humans, in which a rise in plasma triglyceride level was observed 2 and 4 hours after a preload- 
A

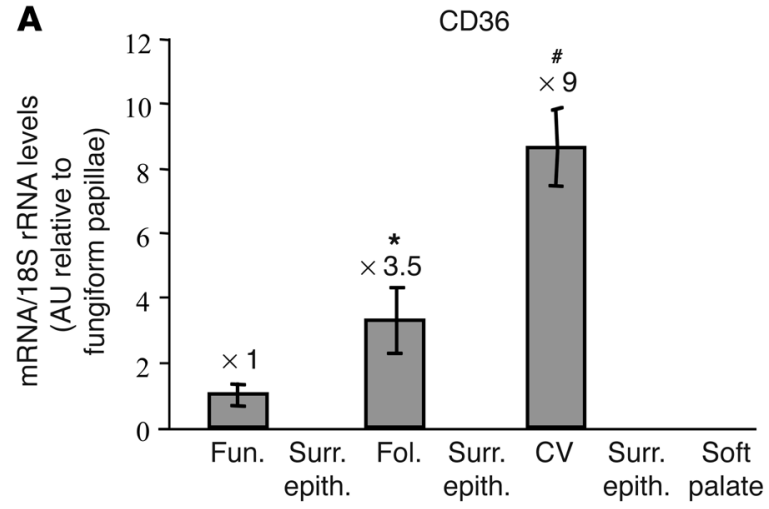

B

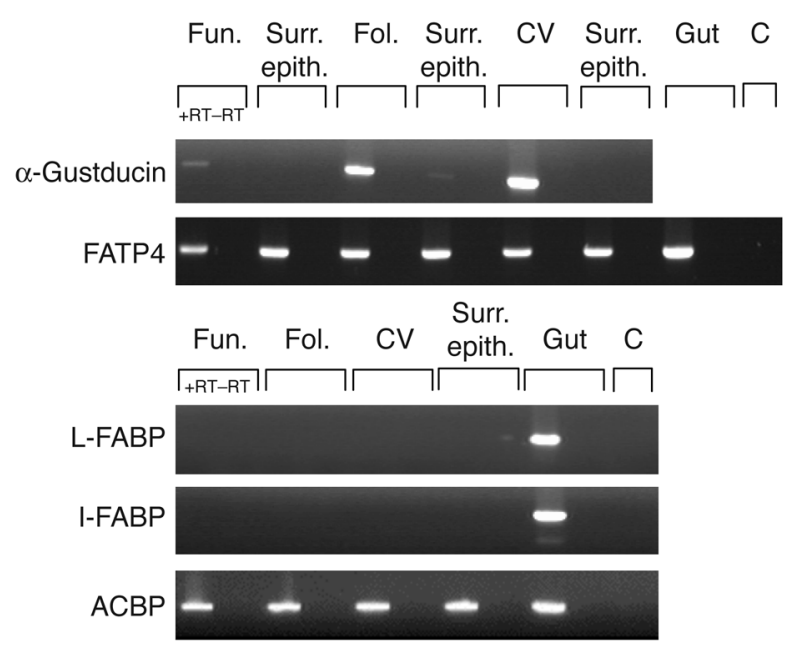

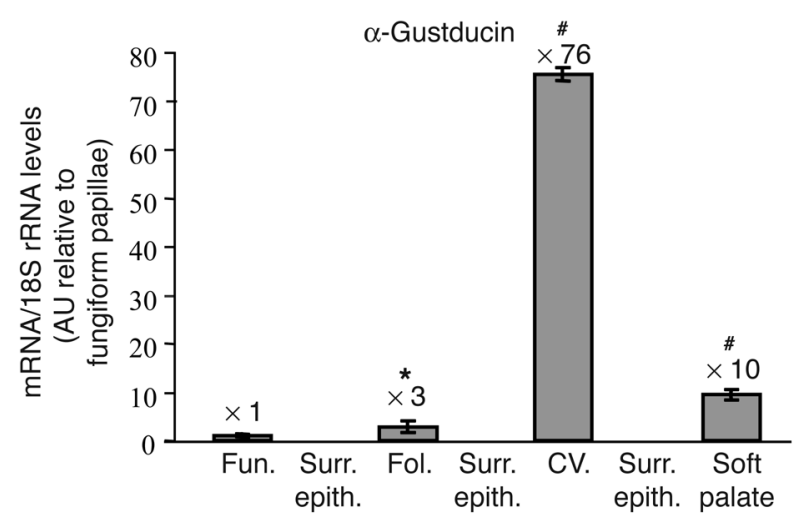

Soft palate

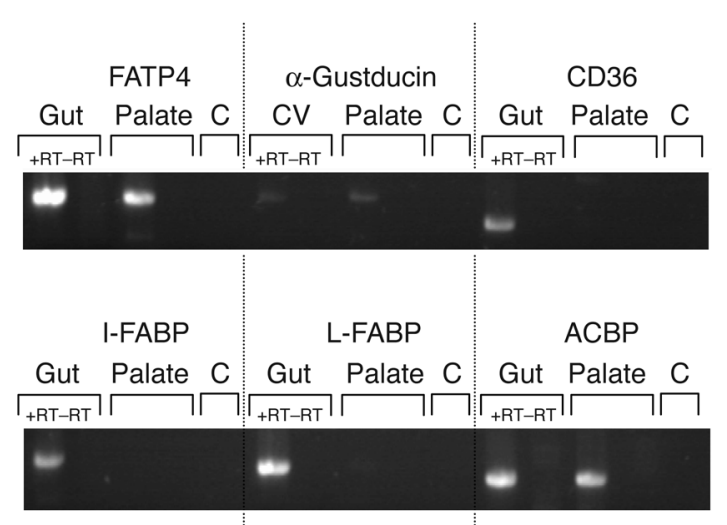

Figure 1

Expression pattern of various lipid-binding proteins and $\alpha$-gustducin throughout the tongue and palatal epithelium in the mouse. Circumvallate, foliate, and fungiform papillae and respective surrounding nongustatory epithelium (negative control) were isolated under a microscope, and total RNA was prepared as described in Methods. (A) Quantification of CD36 and $\alpha$-gustducin mRNA by the SYBR Green method. Each value corresponds to a pool of total RNA from 3-5 mice. Data represent mean + SEM; $n=5$. ${ }^{*} P<0.05$; $P<0.001$. The expression levels relative to fungiform papillae are indicated above the bars. (B) RT-PCR analysis of $\alpha$-gustducin, CD36, FATP-4, L-FABP, I-FABP, and ACBP mRNA. Each experiments is representative of 3 independent experiments performed on a pool of total RNA from 3-5 mice. C, control; CV, circumvallate papillae; Fol., foliate papillae; Fun., fungiform papillae; +RT-RT, RT-PCR without reverse transcriptase; Surr. epith., surrounding nongustatory epithelium.

ing with encapsulated oil (to avoid oral lipid exposure) followed by mastication and expectoration of lipid-enriched foods. This effect was lipid dependent, since no change was noticed with a lipid-free sham feeding (20).

Although all these observations argue in favor of a taste for fat, the nature and physiological function(s) of an oral lipid sensor remain elusive. In the rat, CD36 appears to be a plausible candidate for this function. To explore this hypothesis, experiments were conducted both in rats and in wild-type and CD36-null mice. As the sensitivity to basic tastes is species specific (21), expression of CD36 in papillae and surrounding nonsensory epithelium was first investigated and compared in mouse and rat. Then, impact of CD36 gene inactivation on short- and long-term fat preference and digestive secretions was explored. Data reported herein provide what we believe to be the first identification of a lipid sensor component in the oral cavity by demonstrating that lingual stimulation of CD36 by unsaturated fatty acids impacts both behavioral and digestive physiology.

\section{Results}

CD36 is specifically found at the apical side of lingual taste bud cells. In mammals, taste buds are found within papillae throughout lingual and palatal mucosa. They are especially concentrated in foliate and circumvallate papillae, respectively localized at the lateral and back sides of the tongue, and weakly present in fungiform papillae on the anterior tip of the lingual mucosa. In rat, CD36 has been found in circumvallate papillae $(11,12)$, raising the possibility of its involvement in the detection of dietary lipids. To explore whether CD36 expression was restricted to the oral sensory epithelium, papillae and the surrounding nonsensory epithelium were isolated from mouse lingual and palatal mucosa and analyzed by conventional and real-time RT-PCR. Oral cavity mapping of the expression pattern of lipid-binding proteins classically found in the digestive tract was also undertaken to determine whether CD36 exhibits specific oral localization as compared with other lipid-binding proteins. Presence of taste buds in the preparations was assessed using $\alpha$-gust- 


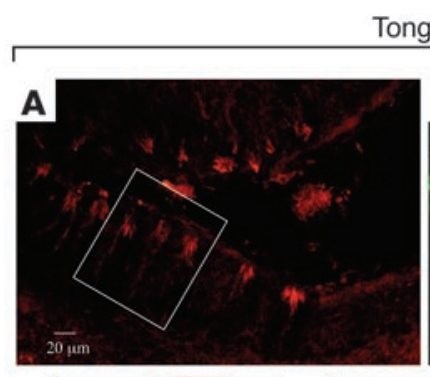

ongue

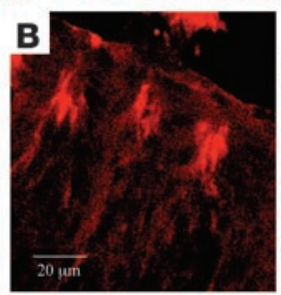

CD36

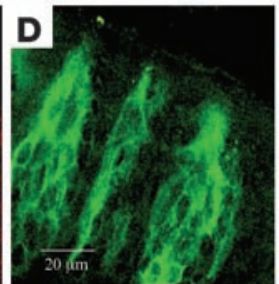

$\alpha$-Gustducin
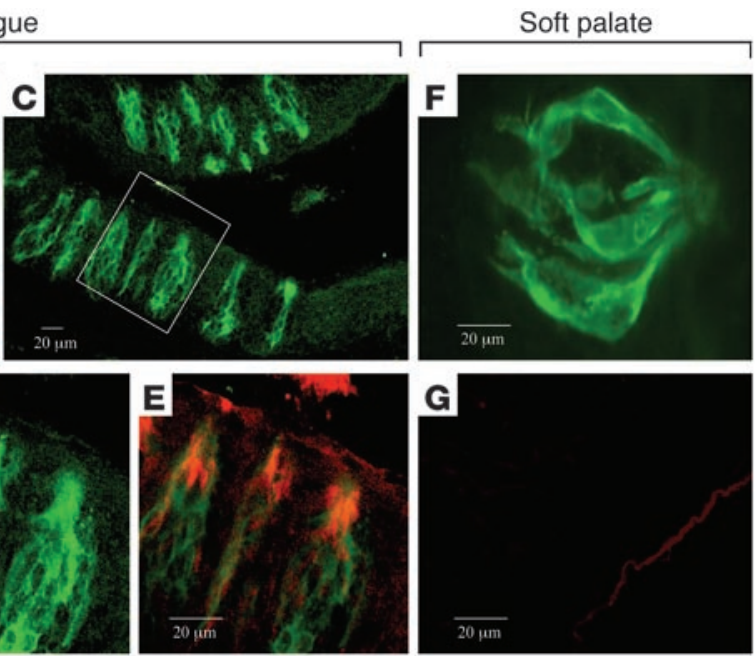

G

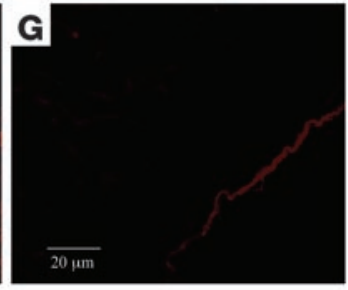

\section{Figure 2}

Immunolocalization of CD36 and $\alpha$-gustducin in the mouse circumvallate papillae and palate. CD36 immunoreactivity was confined to the apical side of taste bud cells in the lingual epithelium (A and $\mathbf{B}$ ), while $\alpha$-gustducin was found throughout the taste buds in spindle-shaped cells (C and D). (E) Coexpression of CD36 and $\alpha$-gustducin was found in some lingual taste receptor cells. Palatal taste buds were positive for $\alpha$-gustducin (F) but negative for CD36 (G). ducin gene expression, since this G protein is involved in the transduction of bitter and sweet tastes (22).

In the mouse, CD36 was strictly restricted to the lingual gustatory papillae, where its expression pattern correlates quite well with that of $\alpha$-gustducin. Indeed, FAT/CD36 was highly expressed in circumvallate papillae, to a lesser extend in foliates, and rarely in fungiform papillae (Figure 1A). In contrast, CD36 was not found in palate, despite the presence of $\alpha$-gustducin (Figure 1A). This was also true in the rat (data not shown). The CD36 expression pattern was not shared by any of the other lipid-binding proteins tested (Figure 1B). Indeed, fatty acid transport protein-4 (FATP-4), a plasma membrane molecule with an acyl-CoA synthetase-like activity (23), and acylCoA-binding protein (ACBP), a housekeeping gene involved in cellular acyl-CoA trafficking (24), were found throughout the lingual and palatal mucosa. As expected, liver fatty acid-binding protein (L-FABP) and intestinal fatty acid-binding protein (I-FABP), which have previously been found to be expressed in tissues characterized by a high LCFA requirement (25), were undetectable in the oral mucosa (Figure 1B). Immunolocalization of
CD36 in the mouse circumvallate papillae revealed a specific staining of the apical side of some taste bud cells lining the taste pores (Figure 2, A and B). Immunoreactivity for $\alpha$-gustducin was found in a large number of taste receptor cells (Figure 2, $\mathrm{C}$ and $\mathrm{D}$ ). Taste receptors cells were unstained when the primary antibodies were absent (data not shown). In agreement with the real-time RT-PCR data (Figure 1A), no CD36 staining was detected in palatal papillae, despite the presence of several spindle-shaped cells immunoreactive for $\alpha$-gustducin (Figure 2, $\mathrm{F}$ and $\mathrm{G})$. Immunostaining analysis of mouse circumvallate papillae indicated that about $78 \%$ of $\alpha$-gustducin positive taste buds were also immunopositive for CD36 $(n=72)$. In a taste bud slice, the number of cells positive for $\alpha$-gustducin and CD36 was $8.0 \pm 2.9$ and $3.0 \pm 0.9$ cells $(n=150)$, respectively. Since an average of 20 cells/slice were counted using Hoechst nuclear staining, it can be estimated that about $40 \%$ of cells were positive for $\alpha$-gustducin and $16 \%$ for CD36. It is important to note that the counting of CD36-positive cells was somewhat challenging due to the apical labeling of the cells. Therefore, it is difficult to accurately estimate the proportion of $\alpha$-gustducin cells that
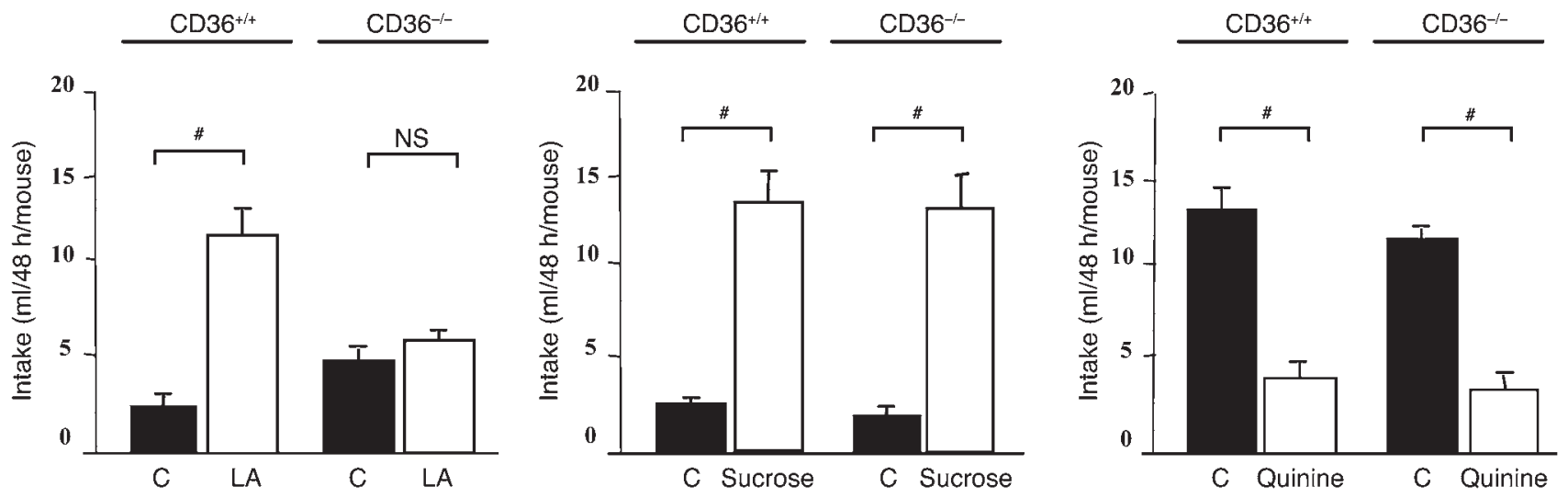

Figure 3

CD36 gene inactivation suppresses the spontaneous lipid preference in mice subjected to 48-hour 2-bottle preference test. Comparison of fluid intake in wild-type and FAT/CD36-null mice. Xanthan gum was used to emulsify linoleic acid (LA) in water and to mimic the lipid texture. The control solutions were water alone or with xanthan gum added. Data represent mean $\pm \mathrm{SEM} ; n=12 .{ }^{\#} P<0.001$. 

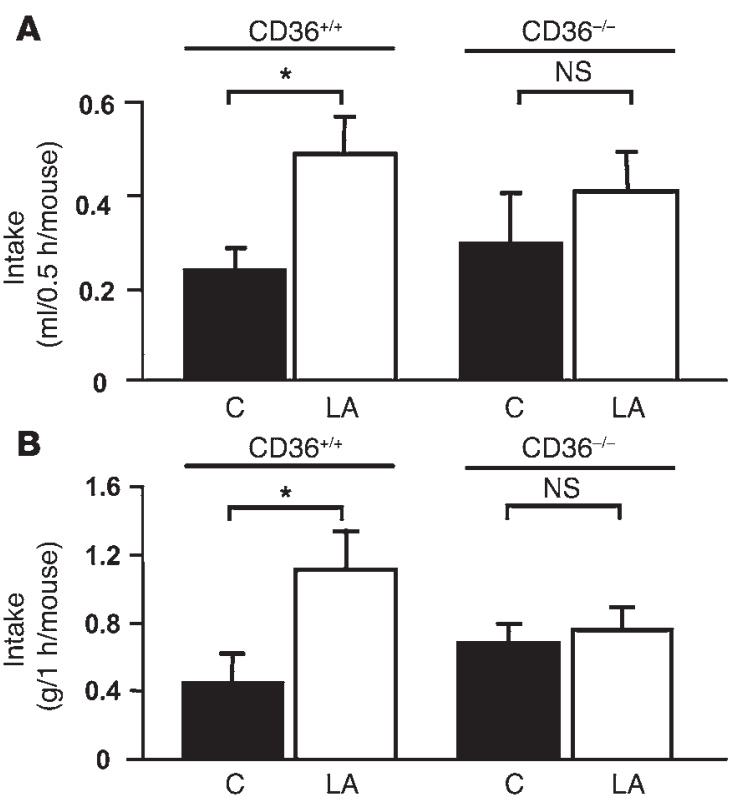

might also have been positive for CD36. Nevertheless, careful analysis of double-immunostained sections suggested that CD36 might be coexpressed with $\alpha$-gustducin in some receptor cells (Figure 2E).

\section{Figure 4}

The short-term preference for lipid-enriched beverages and meals found in the wild-type mice is CD36 dependent. (A) Fluid intake in 1hour-water-restricted wild-type and FAT/CD36-null mice subjected for 0.5 hours to a 2-bottle preference test. Xanthan gum (0.3\%) was used to emulsify $2 \%$ linoleic acid in water and to mimic the lipid texture. The control solution was water with $0.3 \%$ xanthan gum added. (B) Food intake in 12-hour-fasted wild-type and FAT/CD36-null mice subjected to a choice between a $5 \%$ linoleic acid- or paraffin oil-enriched diet for 1 hour. ${ }^{*} P<0.05$.

Invalidation of $C D 36$ gene abolishes the spontaneous preference for LCFAs. To determine whether CD36 plays a role in the oral detection of lipids, wild-type and CD36-null mice were subjected to 2-bottle preference tests. Wild-type mice exhibited a strong preference for a $2 \%$ linoleic acid-enriched solution as compared with a control solution containing $0.3 \%$ xanthan gum to emulsify the fatty acid and mimic lipid texture (Figure 3 ). Similar results were reproduced with $10 \%$ linoleic acid (data not shown). Such a linoleic acid-mediated effect is not species specific, since it has been already found in the rat (7). In contrast, CD36-null mice did not discriminate between the control and linoleic acid-enriched solutions (Figure 3). This loss of preference is strictly limited to the lipid detection, since wild-type and CD36-null animals exhibited a similar response for sucrose or quinine solutions (Figure 3). To explore whether this stereotypic behavior observed with long-term (48-hour) preference tests occurred independently of
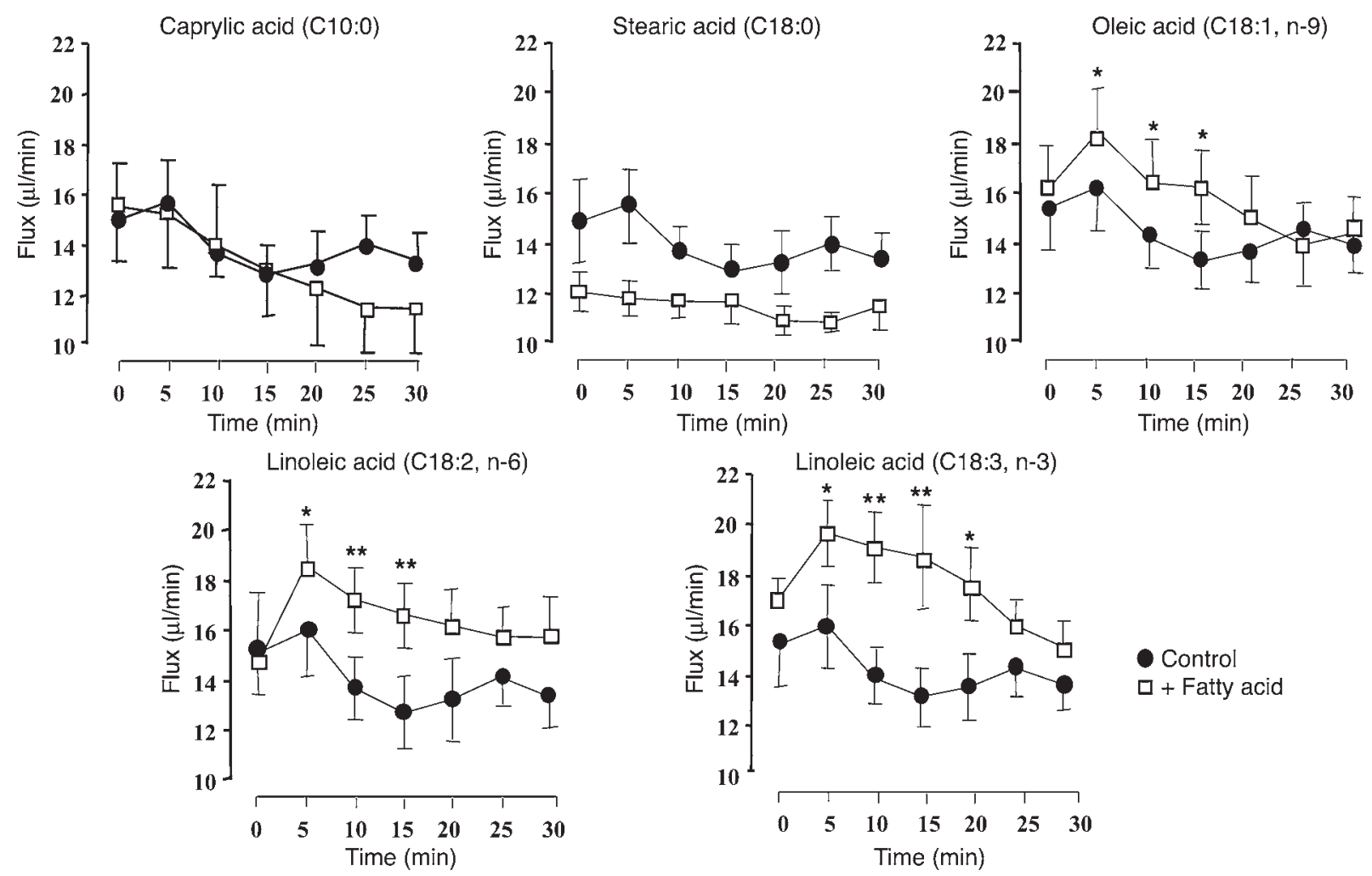

Figure 5

Effect of lingual fatty acid load on bile flux in rats. Anesthetized rats with bile diversion and esophageal ligation to prevent any lipid ingestion were subjected to an oral load of different purified fatty acids $(0.2 \mathrm{ml})$. Controls received $0.2 \mathrm{ml}$ water by the same route. Filled circles and open squares correspond to control solution and fatty acids tested, respectively. Data represent mean $\pm \mathrm{SEM} ; n=9$. ${ }^{*} P<0.05 ;{ }^{* \star} P<0.01$. 

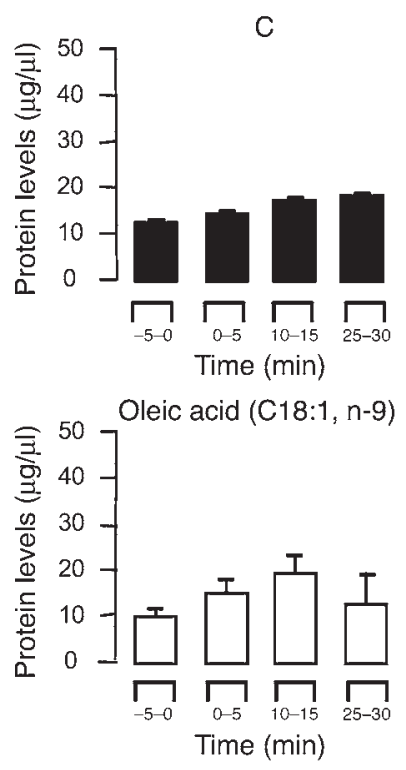

postingestive cues, water-restricted mice were subjected for 0.5 hours to a 2-bottle test. As shown in Figure 4A, wild-type mice drank about 2 -fold more linoleic acid solution than the control beverage containing xanthan gum. However, this fatty acid preference was not reproduced in CD36-null mice. Preference tests were next extended to a standard meal, with mice subjected to an optional ingestion of 2 diets with different LCFA contents. Results showed that CD36-null mice displayed a lower preference for solid diets enriched with LCFAs compared with wildtype controls (Figure 4B).

The changes in pancreatobiliary secretions mediated by oral delivery of fatty acids are CD36 dependent. A potential physiological advantage of an oral lipid detection is the functional preparation of the digestive tract to incoming lipid. This assumption is supported by a recent report showing that an oral lipid load was sufficient to enhance the protein content of pancreatobiliary juice in esophagostomized rats (18). To further explore this hypothesis, the effects of an oral load with different types of fatty acids on pancreatobiliary flux and composition were assessed in rats with an esophageal ligation to prevent nutrient ingestion. As shown in Figure 5, a rapid rise in bile flux occurred following oleic acid, linoleic acid, or linolenic acid deposition in the oral cavity. This effect was highly dependent on both the length of carbon chain and the presence of 1 or several double bonds, since it was not reproduced with medium-chain and saturated LCFAs. If no change in pancreatic flux was observed (e.g., $10.0 \pm 2.1$ vs. $7.4 \pm 1.0 \mu \mathrm{l} / 30 \mathrm{~min} / \mathrm{rat}$ in controls after linoleic acid load onto lingual epithelium; $n=9$; $P=$ NS, a strong increase in the protein content of pancreatic juice was observed in rats stimulated by polyunsaturated fatty acids (Figure 6). The contribution of CD36 to these effects was assessed using intact and CD36-null mice with esophageal ligation and a pancreatobiliary catheter. As shown in Figure 7, the linoleic acid-mediated induction of both flux and protein content of pancreatobiliary secretions found in wild-type mice was fully abolished in CD36-null mice. Similar data were obtained in rats: the rise in bile flux triggered by the direct deposition of linoleic acid onto the tongue $(561.4 \pm 39.7$ vs. $442.8 \pm 73.0 \mu \mathrm{l}$ bile $/ 30 \mathrm{~min} / \mathrm{rat}$ in control; $P<0.05 ; n=9$ ) was not observed when
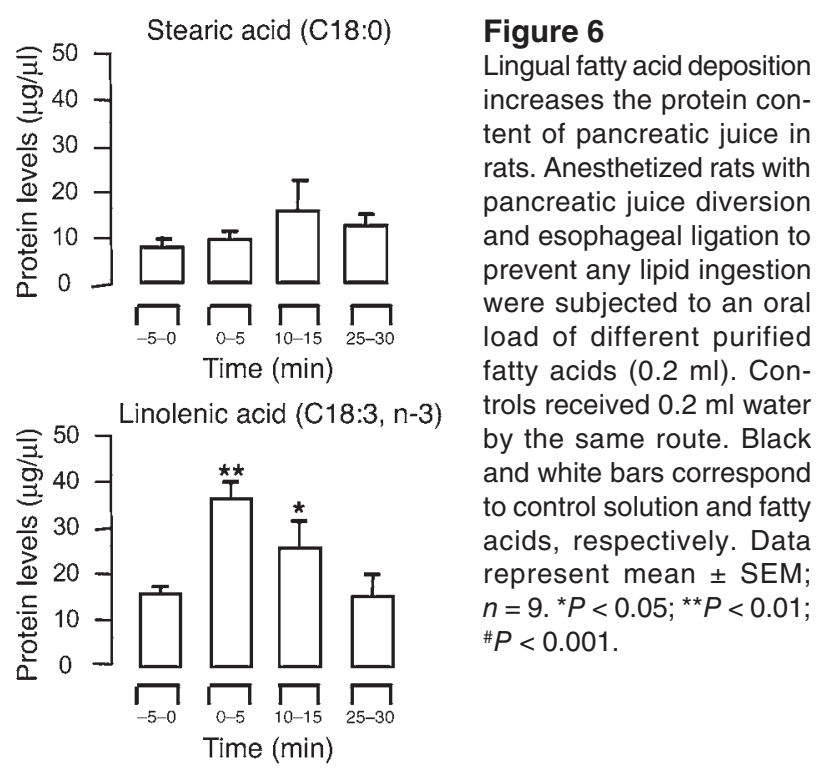

this fatty acid was applied onto the soft palate, i.e., onto a CD36negative sensory mucosa $(512.6 \pm 66.0$ vs. $527.4 \pm 73.0 \mu \mathrm{l} \mathrm{bile/30}$ $\mathrm{min} / \mathrm{rat}$ in control; $n=5 ; P=\mathrm{NS}$ ).

\section{Discussion}

Recent studies strongly suggest the involvement of gustation in the spontaneous preference for lipids both in rats and mice $(7,26)$. Herein, we report the first demonstration to our knowledge of the crucial role played by the integral membrane glycoprotein CD36 in this phenomenon. Indeed, CD36 deficiency fully abolished the high palatability of the LCFA-enriched solutions observed in wild-type mice. This effect was specifically restricted to lipid detection, since the preference for sucrose and the aversion for quinine remained unchanged in wild-type and CD36-null mice. This behavioral specificity might be accounted for by involvement of CD36 in a specialized lipid detection system localized within the oral cavity and/or by postingestive stimuli leading to an increase in lipid intake. Short-term preference tests and anatomical expression within the mouse oral mucosa supported the role of CD36 as a specific oral lipid sensor. Indeed, wild-type mice subjected to a 2-bottle test for only 0.5 hours displayed a higher preference for LCFA-enriched solution than did CD36-null mice. This experimental design excluding postingestive cues highlights the role of CD36 in the preference for fat. Moreover, in circumvallate papillae, CD36 expression appears to be restricted to cells lining the taste pores. Such an apical localization in papillae known to have the highest taste bud density is particularly adaptive to generate a fat stimuli. Indeed, CD36 is directly exposed to an extracellular medium enriched in LCFAs as a result of locally increased lingual lipase levels (8). These data are also in good agreement with our previous observation that CD36 is confined to the apical microvilli of enterocytes (27). The role of CD36 as lipid sensor is further supported by its predicted protein structure. CD36 has a large extracellular lipid-binding pocket and an intracellular tail that has previously been shown to be associated with kinases (15-17). Additional studies are required to determine whether the interaction between LCFAs and CD36 can transduce a signal at the origin of fat taste. An alternative possibility might be that LCFAs are direct- 

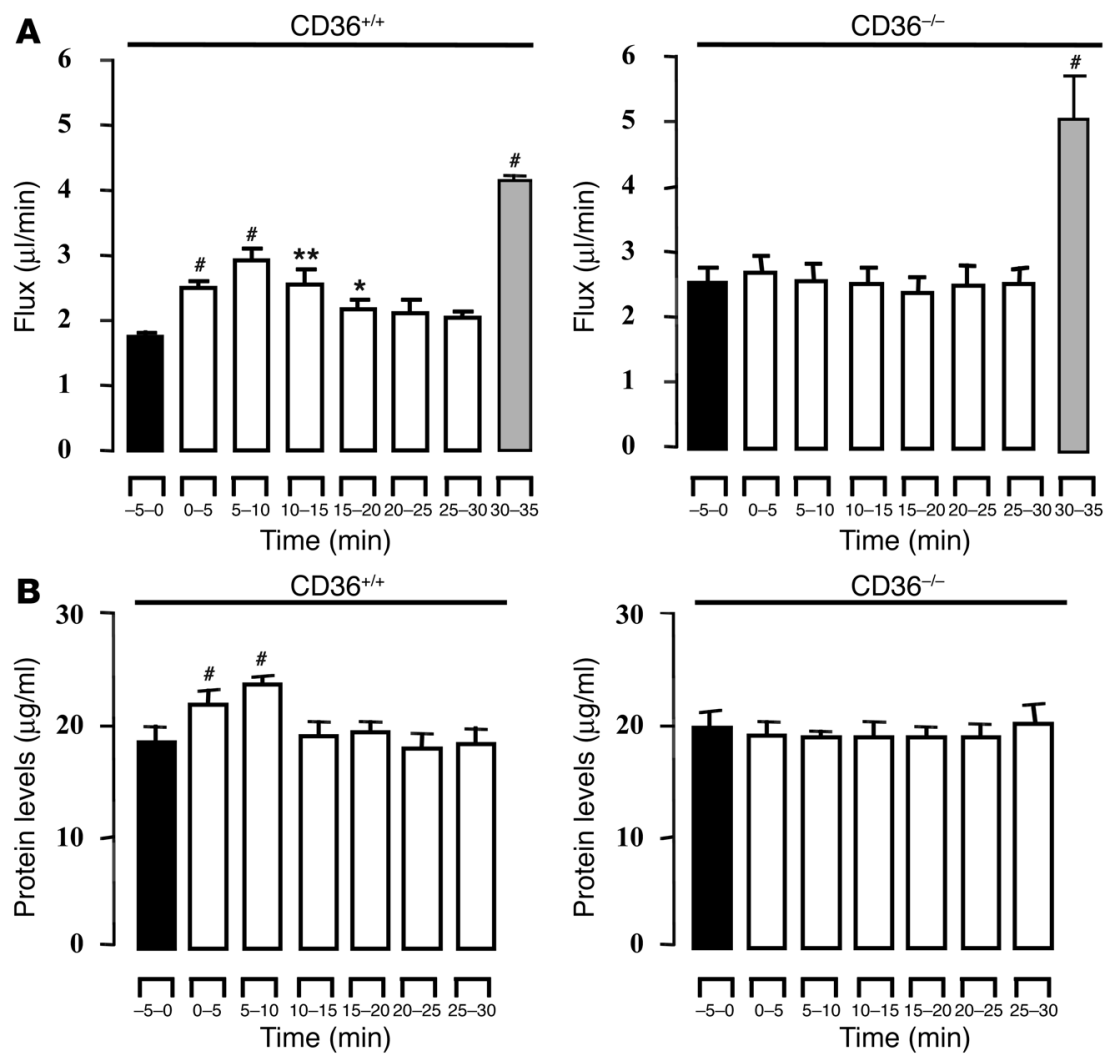

Figure 7

Impact of CD36 gene invalidation on the changes in pancreato-biliary flux (A) and protein levels (B) triggered by a lingual linoleic acid deposition. Anesthetized wild-type and CD36-null mice with pancreato-biliary diversion and esophageal ligation to prevent any lipid ingestion were subjected to an oral load with linoleic acid $(0.2 \mathrm{ml})$. Controls received $0.2 \mathrm{ml}$ water by the same route. Black and white bars correspond to pancreato-biliary flux before and after lingual linoleic acid deposition, respectively. The functional validity of preparations was assessed by a duodenal $\mathrm{HCl}$ infusion (gray bars). Data represent mean $\pm \mathrm{SEM} ; n=9$. ${ }^{\star} P<0.05$; ${ }^{* *} P<0.01 ;{ }^{\#} P<0.001$.

ly delivered by $\mathrm{CD} 36$ to delayed rectifying $\mathrm{K}^{+}$channels found in taste receptor cells and responsible for transduction of some taste stimuli (9). Since in vitro unsaturated fatty acids inhibit $\mathrm{K}^{+}$currents only when they are applied extracellularly (28), CD36 might serve in vivo as a docking site for LCFAs in the plasma membrane of taste receptor cells, facilitating the closure of $\mathrm{K}^{+}$channels leading, in turn, to firing of afferent nerve fibers.

The physiological importance of CD36 in oral lipid detection is further demonstrated by the fact that CD36 gene disruption fully suppressed qualitative and quantitative changes in pancreatobiliary secretions triggered by lingual linoleic acid deposition in intact mice with esophageal ligation. Similar effects were also found in rats. Indeed, the sustained increase in bile flux and protein content of pancreatic juice mediated by lingual linoleic acid deposition were not reproduced when this LCFA was directly loaded onto the palatal mucosa, i.e., onto an area of the mucosa negative for CD36. Once again, postingestive contributions of CD36 can be excluded in these experiments, since the secretory changes occurred in the absence of lipid ingestion. The mechanisms responsible for the perception of primary tastes exhibits a wide species variability (e.g., sourness in rodents; ref. 21). Because expression of CD36 within the oral cavity and its effect on digestive secretions are conserved in mice and rats, it is likely that CD36 performs a basic function in the taste bud cells. The physiological advantage provided by such an orosensory system may be a contribution to the cephalic phase of digestion, leading to the optimization of dietary lipid digestion and absorption by anticipation of their arrival in the small intestine.

CD36 is broadly expressed in many cells/ tissues, has a wide variety of ligands, and thus has physiological roles in cell adhesion, as a scavenger receptor, and in cellular lipid transfer (29). CD36 has been shown to contribute to cellular triglyceride synthesis and peripheral clearance of chylomicrons (30), fatty acid oxidation in muscle, and lipid storage by adipose tissue (for reviews, see refs. 31, 32). The present data strongly suggest that CD36 also plays a role as a LCFA sensor critical for the selection of lipid-rich foods and the cephalic phase of digestive secretions. These complementary functions highlight the importance of $\mathrm{CD} 36$ for an optimal utilization of dietary lipids. The fact that CD36 deficiency shows some features of metabolic syndrome (i.e., dyslipidemia, insulin resistance, hypertension) both in animals models and humans is consistent with this assumption (for a review, see ref. 33).

The sense of taste informs the organism about the quality of ingested food. It is classically depicted in human as being composed of 5 submodalities directed to the perception of sweet, salt, sour, bitter, and umami stimuli. The possibility for an additional modality directed to fat has often been suggested but remains a matter of debate. The results presented herein constitute the first molecular support for the existence of an orosensory receptor for lipids in rodents. Overconsumption of dietary fat greatly contributes to the current epidemic of obesity. Mice to which corn oil was given as an optional supplement to standard laboratory chow over a long period exhibit a chronic excessive caloric intake and develop obesity (34). Our data raise the possibility that CD36-mediated fat perception in food may contribute to the obesity risk. Further experiments are now required to understand the events underlying transduction of LCFA signaling in taste bud cells and to explore the neural pathway affecting behavioral and digestive physiology.

\section{Methods}

Experimental procedures. French guidelines for the use and the care of laboratory animals were followed, and experimental protocols were approved by the animal ethics committee of Burgundy University. Wistar rats and FAT/CD36 wild-type or -null mice (backcrossed 6 times to C57BL/6J mice) (35) were housed individually in a controlled environment (constant temperature and humidity, darkness from $8 \mathrm{pm}$ to $8 \mathrm{am}$ ) and fed ad libitum a standard laboratory chow (UAR A04; Usine d'Alimentation Rationnelle). The strategy used to invalidate CD36 eliminated a portion of introns 2 and 3 and the entire third exon. No regulatory element is known in these intronic sequences (36). In the region of chromosome 5 
where CD36 is located, the nearest genes up- or downstream are about $0.05 \mathrm{Mb}$ away. Additionally, there are no genes or expressed sequence tags overlapping with the CD36 gene.

In a first set of experiments, rats and C57BL/6J wild-type mice (Charles River Laboratories) were used to establish the expression pattern throughout the lingual and palatal epithelia of major lipid-binding proteins found in the digestive tract: CD36 (15), FATP-4 (35), I-FABP and L-FABP (37), and ACBP (38). Fungiform, foliate, and circumvallate papillae were isolated according to previously published procedures $(39,40)$. In brief, lingual epithelium was separated from connective tissue by enzymatic dissociation (elastase and dispase mixture, $2 \mathrm{mg} / \mathrm{ml}$ each in Tyrode buffer: $140 \mathrm{mM} \mathrm{NaCl}, 5 \mathrm{mM}$ $\mathrm{KCl}, 10 \mathrm{mM}$ HEPES, $1 \mathrm{mM} \mathrm{CaCl}_{2}, 10 \mathrm{mM}$ glucose, $1 \mathrm{mM} \mathrm{MgCl}_{2}, 10 \mathrm{mM}$ Na pyruvate, $\mathrm{pH}$ 7.4) and papillae dissected under a microscope. Epithelium surrounding the papillae was also collected to serve as nonsensory control tissue. Palatal mucosa was isolated by peeling. Intestinal mucosa were used as positive control for lipid-binding proteins. Samples were snap-frozen in liquid nitrogen and stored at $-80^{\circ} \mathrm{C}$ until RNA extraction.

In a second set of experiments, the effect of $\mathrm{CD} 36$ gene invalidation on the spontaneous preference for lipid-enriched solutions $(7,26)$ was assessed using the 2-bottle preference test. After a training period of 3 days, individually caged wild-type and CD36-null mice were subjected for 2 days to a choice between $2 \%$ purified linoleic acid emulsified in $0.3 \%$ xanthan gum in water or water with vehicle $(0.3 \%$ xanthan gum) alone. The left-right position of the 2 bottles was switched daily to avoid side preference. The fluid intake was measured and expressed as milliliters/48 hours/mouse. To determine the specificity of the effect, we used $4 \%$ sucrose and $300 \mu \mathrm{M}$ quinine solutions in control experiments. Mice received water alone for 3 days between experimental periods. To exclude postingestive interference, short-term ( 0.5 hours) 2 -bottle preference tests with $2 \%$ linoleic acid were also performed in water-restricted $\mathrm{CD} 36^{+/+}$and $\mathrm{CD} 36^{-/-}$mice for 1 hour prior the experiment. Finally, the behavioral study was extended to solid diet. Individually caged animals were trained to eat reconstituted semisynthetic laboratory chow dispensed in 2 similar containers. Before being subjected to a choice between a $5 \%$ linoleic acid- or paraffin oil-enriched diet for 1 hour, mice were fasted for 12 hours.

The third set of experiments was undertaken to evaluate the impact of a fatty acid deposition in the oral cavity on digestive secretions. Fasted rats and wild-type and CD36-null mice were anesthetized with sodium pentobarbital ( $0.1 \mathrm{ml} / 100 \mathrm{~g}$ body mass), and the esophagus was ligated to avoid nutrient ingestion. After a laparotomy, a double catheterization of the pancreatobiliary duct was performed in rats to collect separately the bile (upper catheter) and the pancreatic juice (lower catheter). In mice, due to the small size of the pancreatobiliary duct, a single catheter was placed near the connection between the duct and the duodenum. After determination of basal fluxes, digestive juice was collected every 5 minutes for 30 minutes following oral administration of free fatty acids $(0.2 \mathrm{ml})$ or control fluid $(0.2 \mathrm{ml}$ water). Purified free fatty acids (SigmaAldrich) tested were caprylic acid (C10:0), stearic acid (C18:0), oleic acid (C18:1, n-9), linoleic acid (C18:2, n-6), or linolenic acid (C18:3, n-3). Protein levels in pancreatic juice were assayed using the bicinchoninic acid (BCA) method (Uptima Kit; Interchim).

RT-PCR. Total RNA from oral epithelium was extracted using RNeasy mini-columns (QIAGEN) and $1 \mu \mathrm{g}$ reverse-transcribed into cDNA using Omniscript reverse transcriptase (200 U; QIAGEN) in a 20- $\mu \mathrm{l}$ reaction volume containing $\times 1$ Omniscript buffer (QIAGEN), $0.5 \mathrm{mM}$ dNTP, $1 \mu \mathrm{M}$ oligo-dT primer (for conventional RT-PCR) or $10 \mu \mathrm{M}$ random hexamers (for real-time RT-PCR), and $10 \mathrm{U}$ of RNase inhibitor. After incubation for 60 minutes at $37^{\circ} \mathrm{C}$, the reaction was stopped by heating $(5$ minutes at $95^{\circ} \mathrm{C}$ ). The expression level of CD36 was determined throughout lingual and palatal epithelia by real-time RT-PCR (ABI PRISM 7700; Applied
Biosystems). $\alpha$-Gustducin, a $G$ protein considered as a molecular marker specific for taste bud cells, was also assayed to assess the purity of papillae samples. RNA levels were normalized against $18 \mathrm{~S}$ ribosomal RNA. Primer/ probe sets were designed with Primer Express software 1.00A (Applied Biosystems) using gene sequences from the GenBank database. Optimized PCR consisted of 40 cycles at $95^{\circ} \mathrm{C}$ for 15 seconds followed by amplification at $60^{\circ} \mathrm{C}$ for 30 seconds. PCR amplification was done using SYBR Green PCR Master Mix (Applied Biosystems) and the following primer sequences: FAT/ CD36 (forward, 5'-GATGACGTGGCAAAGAACAG-3'; reverse, 5' -TCCTCGGGGTCCTGAGTTAT- ${ }^{\prime}$ ), $\alpha$-gustducin (forward, $5^{\prime}$ GAGAGCAAGGAATCAGCCAG-3'; reverse, 5'-GTGCTTTTCCCAGATTCACC-3'), 18S ribosomal RNA (forward, 5'-TAAGTCCCTGCCCTTGGTACACA-3'; reverse, $5^{\prime}$-GATCCGAGGGCCTCACTAAAC-3'). The comparative $2^{-\Delta \Delta C T}$ method (41) was used to determine CD36 and $\alpha$-gustducin expression levels.

Comparison of expression pattern of CD36 with other lipid-binding proteins (FATP-4, L-FABP, I-FABP, and ACBP) was determined by RT-PCR amplification using a Whatman Biometra thermocycler and agarose gel electrophoresis. Stock PCR reaction mixtures $(20 \mu \mathrm{l})$ were prepared on ice and contained $0.1 \mu \mathrm{g}$ cDNA, $1.8 \mu \mathrm{ldNTP}(2.5 \mathrm{mM} / \mathrm{dNTP}), 2 \mu \mathrm{l} \times 10 \mathrm{PCR}$ buffer, $0.5 \mu \mathrm{l}$ each primer $(30 \mu \mathrm{M})$, and $0.3 \mu \mathrm{l}$ Taq polymerase (1,000 U; QIAGEN). Amplification cycles consisted of 30 seconds at $94^{\circ} \mathrm{C}, 30$ seconds at the specific melting temperature of each probe, and 1 minute at $72^{\circ} \mathrm{C}$. The following primers were used: FATP-4 (forward, $5^{\prime}$-AAAAGGAGCTGCCTCTG-3'; reverse, 5'-AAGGAGCCTATCAGAAACC-3'), L-FABP (forward, 5'-GGAAAGGAAACCTCATTG-3'; reverse, 5'-CCTTGTCTAAATTCTCTTGC-3'), I-FABP (forward, 5'-TCCTAGAGACACACACAG-3', reverse, 5'-CTTAGCTCTTCAGCGTTG-3'), ACBP (forward, 5'-CTTGATTGCTCCTGCTTC-3'; reverse, 5'-GGCATTATGTCCTCACAG-3'), CD36 (forward, 5'-TCATTGCAACTGAGTGG-3'; reverse, 5' - TCATAAGCTCTGTGTCAG- $3^{\prime}$ ), and $\alpha$-gustducin (forward, $5^{\prime}$-AAGCTTCAGGAGGATGC3'; reverse, 5'-AAAGGAGAATTGAGTTTCAATG-3').

Immunohistochemistry. Excised circumvallate and palatal papillae were embedded in OCT medium (Tissue-Tek; Oxford Instruments) and snapfrozen in isopentane chilled with liquid nitrogen. Cryostat sections $(14 \mu \mathrm{m})$ were air dried for 2 hours at room temperature, fixed in $95 \%$ ethanol for 5 minutes, and rehydrated in $0.1 \mathrm{M} \mathrm{PBS}$ ( $\mathrm{pH} 7.4$ ) for 10 minutes. Rehydrated sections were blocked in $5 \%$ goat serum (Sigma-Aldrich) and $0.2 \%$ Triton $\mathrm{X}-100$ in PBS for 15 minutes at room temperature and incubated overnight at $4{ }^{\circ} \mathrm{C}$ with a $1: 100$ dilution of the anti-mouse CD36 antibody UA009 (12) and with a commercial polyclonal anti-mouse $\alpha$-gustducin antibody (1:150; Santa Cruz Biotechnology Inc.) raised in rabbit. After washing, sections were next incubated for 2 hours at room temperature with labeled secondary antibodies (1:600 dilution of Cy3-conjugated anti-mouse IgG and FITC-conjugated anti-rabbit IgG from Sigma-Aldrich). Staining specificity was assessed by treating slices in the absence of primary antibodies. For counting cells, slices were counterstained with Hoechst reactive $(0.1 \mathrm{mg} / \mathrm{ml}$; Sigma-Aldrich) in the mounting media to determine the number of cells. Slices were analyzed either under a confocal microscope (Leica TCS4D) or by transmission (Zeiss Axioskop with AxioCam MRc5).

Statistics. Results are expressed as mean \pm SEM. The significance of difference between groups was evaluated by ANOVA or 2-tailed Student's $t$ test. A $P$ value of less than 0.05 was considered to be statistically significant.

\section{Acknowledgments}

We thank Graham Mayrhofer (Department of Molecular Biosciences, University of Adelaide, Australia) for the generous gift of the mouse FAT/CD36 antibody. We would also like to thank A. Holley, P. Macleod, and B. Schaal for critical reading of the manuscript and M.-C. Monnot for her excellent technical assistance. This work was supported by the National Institute of Agronomic 
Research (INRA) through the Research Program in Human Nutrition (PRNH to P. Besnard).

Received for publication April 8, 2005, and accepted in revised form August 23, 2005.

1. Drewnowski, A., Brunzell, J.D., Sande, K., Iverius, P.H., and Greenwood, M.R. 1985. Sweet tooth reconsidered: taste responsiveness in human obesity. Physiol. Behav. 35:617-622.

2. Mela, D.J., and Sacchetti, D.A. 1991. Sensory preferences for fats: relationships with diet and body composition. Am. J. Clin. Nutr. 53:908-915.

3. French, S., and Robinson, T. 2003. Fats and food intake. Curr. Opin. Clin. Nutr. Metab. Care. 6:629-634.

4. Mela, D.J. 1988. Sensory assessment of fat content in fluid dairy products. Appetite. 10:37-44.

5. Takeda, M., Sawano, S., Imaizumi, M., and Fushiki, T. 2001. Preference for corn oil in olfactory-blocked mice in the conditioned place preference test and the two-bottle choice test. Life Sci. 69:847-854.

6. Fukuwatari, T., et al. 2003. Role of gustation in the recognition of oleate and triolein in anosmic rats. Physiol. Behav. 78:579-583.

7. Tsuruta, M., Kawada, T., Fukuwatari, T., and Fushiki, T. 1999. The orosensory recognition of long-chain fatty acids in rats. Physiol. Behav. 66:285-288.

8. Kawai, T., and Fushiki, T. 2003. Importance of lipolysis in oral cavity for orosensory detection of fat. Am. J. Physiol. 285:R447-R454.

9. Gilbertson, T.A., Fontenot, D.T., Liu, L., Zhang, H., and Monroe, W.T. 1997. Fatty acid modulation of $\mathrm{K}+$ channels in taste receptor cells: gustatory cues for dietary fat. Am. J. Physiol. 272:C1203-C1210.

10. Gilbertson, T.A. 1998. Gustatory mechanisms for the detection of fat. Curr. Opin. Neurobiol. 8:447-452.

11. Fukuwatari, T., et al. 1997. Expression of the putative membrane fatty acid transporter (FAT) in taste buds of the circumvallate papillae in rats. FEBS Lett. 414:461-464.

12. Zhang, X., et al. 2003. CD36/fatty acid translocase in rats: distribution, isolation from hepatocytes, and comparison with the scavenger receptor SR-B1. Lab. Invest. 83:317-332.

13. Baillie, A.G., Coburn, C.T., and Abumrad, N.A. 1996. Reversible binding of long-chain fatty acids to purified FAT, the adipose CD36 homolog. J. Membr. Biol. 153:75-81.

14. Ibrahimi, A., et al. 1996. Expression of the CD36 homolog (FAT) in fibroblast cells: effects on fatty acid transport. Proc. Natl. Acad. Sci. U. S. A. 93:2646-2651.

15. Abumrad, N.A., el-Maghrabi, M.R., Amri, E.Z., Lopez, E., and Grimaldi, P.A. 1993. Cloning of a rat adipocyte membrane protein implicated in binding or transport of long-chain fatty acids that is induced during preadipocyte differentiation. Homology with human CD36. J. Biol. Chem.

Address correspondence to: Philippe Besnard, Physiologie de la Nutrition, Ecole Nationale Supérieure de Biologie Appliquée à la Nutrition et à l'Alimentation, 1 Esplanade Erasme, F-21000 Dijon, France. Phone and Fax:33-380-396-691; E-mail: pbesnard@ u-bourgogne.fr.

268:17665-17668.

16. Greenwalt, D.E., et al. 1992. Membrane glycoprotein CD36: a review of its roles in adherence, signal transduction, and transfusion medicine. Blood. 80:1105-1115

17. Huang, M.-M., Bolen, J.B., Barnwell, J.W., Shattil, S.J., and Brugge, J.S. 1991. Membrane glycoprotein IV (CD36) is physically associated with the fyn, lyn, and yes protein-tyrosine kinases in human platelets. Proc. Natl. Acad. Sci. U. S. A. 88:7844-7848.

18. Hiraoka, T., Fukuwatari, T., Imaizumi, M., and Fushiki, T. 2003. Effects of oral stimulation with fats on the cephalic phase of pancreatic enzyme secretion in esophagostomized rats. Physiol. Behav. 79:713-717.

19. Ramirez, I. 1985. Oral stimulation alters digestion of intragastric oil meal in rats. Am. J. Physiol. 248:R459-R463.

20. Mattes, R.D. 2001. The taste of fat elevates postprandial triacylglycerol. Physiol. Behav. 74:343-348.

21. DeSimone, J.A., Lyall, V., Heck, G.L., and Feldman, G.M. 2001. Acid detection by taste receptor cells. Respir. Physiol. 129:231-245.

22. Margolskee, R.F. 2002. Molecular mechanisms of bitter and sweet taste transduction. J. Biol. Chem. 277:1-4.

23. Herrmann, T., et al. 2001. Mouse fatty acid transport protein 4 (FATP4): characterization of the gene and functional assessment as a very long chain acyl-CoA synthetase. Gene. 270:31-40.

24. Knudsen, J., Neergaard, T.B., Gaigg, B., Jensen, M.V., and Hansen, J.K. 2000. Role of acylCoA binding protein in acyl-CoA metabolism and acyl-CoA-mediated cell signaling. J. Nutr. 130(2S Suppl.):294S-298S.

25. Niot, I., and Besnard, P. 2003. Intestinal fat absorption: roles of intracellular lipid-binding proteins and peroxisome proliferator-activated receptors. In Cellular proteins and their fatty acids in bealth and disease. A.K. Duttaroy and F. Spener, editors. WileyVCH. Weinhem, Germany. 359-381.

26. Takeda, M., Imaizumi, M., and Fushiki, T. 2000. Preference for vegetable oils in the two-bottle choice test in mice. Life Sci. 67:197-204.

27. Poirier, H., Degrace, P., Niot, I., Bernard, A., and Besnard, P. 1996. Localization and regulation of the putative membrane fatty-acid transporter (FAT) in the small intestine. Comparison with fatty acid-binding proteins (FABP). Eur. J. Biochem. 238:368-373

28. Gilbertson, T.A. 1998. Gustatory mechanisms for the detection of fat. Curr. Opin. Neurobiol. 8:447-452.
29. Coburn, C.T., and Abumrad, N.A. 2003. Structure-function of CD-36 and evidence for its role in facilitating membrane fatty acid transport. In Cellular proteins and their fatty acids in bealth and disease. A.K. Duttaroy and F. Spener, editors. Wiley-VCH. Weinhem, Germany. 3-30.

30. Drover, V.A., et al. 2005. CD36 deficiency impairs intestinal lipid secretion and clearance of chylomicrons from the blood. J. Clin. Invest. 115:1290-1297. doi:10.1172/JCI200521514.

31. Ibrahimi, A., and Abumrad, N.A. 2002. Role of CD36 in membrane transport of long-chain fatty acids. Curr. Opin. Clin. Nutr. Metab. Care. 5:139-145.

32. Kuang, M., Febbraio, M., Wagg, C., Lopaschuk, G.D., and Dyck, J.R. 2004. Fatty acid translocase/CD36 deficiency does not energetically or functionally compromise hearts before or after ischemia. Circulation. 109:1550-1557.

33. Hirano, K., et al. 2003. Pathophysiology of human genetic CD36 deficiency. Trends Cardiovasc. Med. 13:136-141.

34. Takeda, M., Imaizumi, M., Sawano, S., Manabe, Y., and Fushiki, T. 2001. Long-term optional ingestion of corn oil induces excessive caloric intake and obesity in mice. Nutrition. 17:117-120

35. Febbraio, M., et al. 1999. A null mutation in murine CD36 reveals an important role in fatty acid and lipoprotein metabolism. J. Biol. Chem. 274:19055-19062.

36. Schaffer, J.E., and Lodish, H.F. 1994. Expression, cloning and characterization of a novel adipocyte long-chain fatty acid transport protein. Cell. 79:427-436.

37. Gordon, J.I., et al. 1985. Tissue specific expression and developmental regulation to two genes coding for rat fatty acid-binding proteins. J. Biol. Chem. 260:1995-1998.

38. Knudsen, J., Hojrup, P., Hansen, H.F., and Roepstorff, P. 1989. AcylCoA binding-protein in the rat. Purification, binding characteristics, tissue concentrations and amino acid sequence. Biochem.J. 262:513-519.

39. Miyamoto, T., Miyazaki, T., Okada, Y., and Sato, T. 1996. Whole-cell recording from non-dissociated taste cells in mouse taste bud. J. Neurosci. Methods. 64:245-252.

40. Ruiz, C.J., et al. 2001. Maintenance of rat taste buds in primary culture. Chem. Senses. 26:861-873.

41. Livak, K.J., and Schmittgen, T.D. 2001. Analysis of relative gene expression data using real-time quantitative PCR and the 2(-Delta Delta C(T)) Method. Methods. 25:402-408. 